\title{
Long Noncoding RNA SERTAD2-3 Inhibits Osteosarcoma Proliferation and Migration by Competitively Binding miR-29c
}

\author{
Zhifa Zhang, ${ }^{1, *}$ Jiangjun Liu, ${ }^{2, *, \dagger}$ Yuezhou Wu, ${ }^{3,{ }^{*}}$ Xuelin Zhao, ${ }^{1}$ Yongyu Hao, ${ }^{1}$ Xiangyu Wang, ${ }^{1}$ Chao Xue, \\ Yan Wang, ${ }^{1}$ Rui Zhang, ${ }^{3}$ and Xuesong Zhang ${ }^{1}$
}

Background: Osteosarcoma (OS) is a malignant tumor disease with high morbidity and mortality in children and adolescents. Recently, attention has been focused on the effects of long noncoding RNAs (lncRNAs) on tumor biology. In this study, we identified the role of lnc-SERTAD2-3 in the development of OS.

Materials and Methods: Sixty OS samples and adjacent tissues were collected to determine the relationship between lnc-SERTAD2-3 levels and clinicopathological characteristics. Quantitative real-time PCR (qPCR) was used to measure gene expression levels. A transwell invasion assay, a Cell Counting Kit- 8 assay, and flow cytometry were used to measure cell migration, growth, and apoptosis, respectively. The binding site between the lnc-SERTAD2-3 and miR-29c RNAs was evaluated using a luciferase reporter assay.

Results: The expression of the lnc-SERTAD2-3 was significantly downregulated in OS samples and three OS cell lines (MG-63, U2OS, and Saos-2) compared to normal tissue. Patients with lower levels of lnc-SERTAD23 expression had a more unfavorable prognosis (larger OS size, distant metastasis, and recurrence). Overexpression of lnc-SERTAD2-3 inhibited proliferation and migration, and promoted apoptosis in OS cells. Moreover, we found that lnc-SERTAD2-3 could suppress miR-29c by direct binding. Moreover, reexpression of miR-29c reversed the effect of lnc-SERTAD2-3 on OS cells.

Conclusion: Overall, lnc-SERTAD2-3, an OS suppressor, is involved in the inhibition of OS proliferation and migration by targeting miR-29c.

Keywords: lnc-SERTAD2-3, miR-29c, osteosarcoma, long noncoding RNA

\section{Introduction}

$\mathbf{O}$ steosarcoma (OS) Is the most common bone malignancy (5 out of 1,000,000 children) in childhood and young adults around the world (Ottaviani and Jaffe, 2009). Owing to advanced diagnostic techniques and improvements in therapy, the prognosis of patients with OS has been significantly enhanced. However, the survival of OS patients with recurrence and metastasis remains unsatisfactory. The 5 -year survival rate was only $10 \%$ in patients with distant metastasis (Allison et al., 2012). Therefore, novel and effective biomarkers for diagnosis, prognosis, and treatment are urgently needed.

Long noncoding RNAs (LncRNAs) are a class of nonprotein-coding transcripts (larger than 200 bases) that participate in a variety of physiological and pathological functions (Wang et al., 2018). Increasing evidence suggests that lncRNAs play a critical role in cancer cell proliferation, migration, and apoptosis, and irregular lncRNAs levels are associated with malignancy initiation and development (Sun et al., 2018; de Oliveira et al., 2019; Flippot et al., 2019). Unfortunately, it is difficult to clarify lncRNA-lncRNA, lncRNA-miRNA, and lncRNA-protein networks due to the large number of lncRNAs involved in these processes. Encouragingly, a number of lncRNAs have been demonstrated to exert an important role in OS, including OS initiation, metastasis, and chemoresistance (Li et al., 2017; Smolle and Pichler, 2018). Thus, identifying these lncRNAs as diagnostic or prognostic biomarkers and therapeutic targets may profoundly improve therapeutic efficacy and clinical outcomes for patients with OS.

To date, the molecular mechanism of lncRNAs underlying the development of OS remains unclear. Recently, it has been proposed that lncRNAs exert "sponge-like" effects on

\footnotetext{
${ }^{1}$ Department of Orthopaedics, The PLA General Hospital, Beijing, China.

${ }^{2}$ Department of Orthopaedics, The Affiliated Hospital of Qingdao University, Qingdao University, Qingdao, China. China.

${ }^{3}$ Department of Emergency Medicine, Xiang'an Hospital of Xiamen University, School of Medicine, Xiamen University, Xiamen,

*These authors contributed equally to this work.

${ }^{\dagger}$ Current affiliation: Department of Orthopaedics, Changhai Hospital, Second Military Medical University, Shanghai, China.
} 
miRNA. Mechanistically, lncRNA can reduce miRNA expression by competitive direct binding, which siphons miRNA away from mRNA and subsequently disables the miRNA (Chen et al., 2017). The lncRNA/miRNA/gene axis usually contributes to regulating cancer biology, including OS. Emerging studies indicate that the IncRNA SERTAD1/2/3 families are indicators of malignancy in patients (Martini et al., 2017; Mongre et al., 2019). In this study, we detected the expression of lnc-SERTAD2-3 in OS and adjacent tissue and investigated the effect of lnc-SERTAD2-3 on OS cell proliferation, invasion, and apoptosis.

\section{Materials and Methods}

\section{Patients and OS specimens}

This study (3012018441) was approved by the Hospital Medical Ethics Association of the People's Liberation Army (PLA) General Hospital (Beijing, China). A total of 60 OS specimens and surrounding normal tissues were collected in the Department of Orthopedics, PLA General Hospital from 2010 to 2018. These specimens were quickly frozen in liquid nitrogen and subsequently transferred to $-80^{\circ} \mathrm{C}$ for storage. Furthermore, the clinical characteristics, including age, sex, tumor size, clinical stage, distant metastasis, and recurrence, were obtained for correlation analysis.

\section{Cell culture and cell transfection}

Human OS cells (MG-63, U2OS, and Saos-2) and hFOB1.19 cells were purchased from Shanghai Institutes for Biological Sciences (Shanghai, China) and maintained in Dulbecco's modified Eagle's medium with high glucose and $10 \%$ fetal bovine serum in a cell culture incubator at a temperature $37^{\circ} \mathrm{C}$ with $5 \%$ carbon dioxide. A lnc-SERTAD2-3overexpressing plasmid was established based on the pcDNA3.1 plasmid (Invitrogen, Carlsbad, CA). miR-29c and miR-con were commercially synthesized by Genepharma (Shanghai, China). For cell transfection, the recombinant plasmid (100 ng) and miRNA (20 nM) were transfected using the Lipofectamine 2000 reagent (Life Technologies, Carlsbad, CA) according to the manufacturer's instructions.

\section{RNA extraction and quantitative real-time PCR}

Total RNA was extracted using the TRIzol reagent (Invitrogen) according to the manufacturer's instructions. Complementary DNA was synthesized using PrimeScript reverse transcriptase (Takara, Kyoto, Japan) and an oligo (dT) primer. Quantitative real-time PCR (qPCR) was performed using SYBR Green (Takara) on an ABI7500 (Applied Biosystems, Carlsbad, CA). The relative expression of lncSERTAD2-3 and phosphatase and tensin (PTEN) was normalized to GAPDH, and the relative expression of miR-29c was normalized to U6.

\section{Cell Counting Kit-8}

Cell Counting Kit-8 (CCK-8) (Dojindo Laboratories, Kumamoto, Japan) was used to evaluate cell viability. In brief, $5 \times 10^{3}$ cells/well were seeded into 96-well plates for $0,1,2$, 3 , and 4 days, followed by incubation with CCK -8 at $37^{\circ} \mathrm{C}$ for $4 \mathrm{~h}$. Then, the optimal density was measured with a Bio-Rad 3550 microplate reader at a wavelength of $450 \mathrm{~nm}$.

\section{Transwell invasion assay}

Matrigel-coated Transwell cell culture chambers $(8 \mu \mathrm{m})$ were used to detect cell invasive ability. The cells, at a density of $1 \times 10^{5}$, were mixed to add into the upper chamber, and $500 \mu \mathrm{L}$ of culture medium containing $10 \%$ serum was added into the lower chamber. The Transwell plate was placed in a cell culture incubator at $37^{\circ} \mathrm{C}$ with $5 \%$ carbon dioxide for $24 \mathrm{~h}$. The cells that did not pass through the chamber were wiped from the inner side of the chamber bottom with a cotton swab. The cells that adhered to the pores of the chamber were fixed with methanol, stained with crystal violet, washed with PBS, and photographed. The number of invasive cells was randomly counted in five fields.

\section{Flow cytometry assay}

Cell cycle analysis was performed as described in a previous study (Liu et al., 2019). In brief, cells were fixed with $70 \%$ ethanol at $4^{\circ} \mathrm{C}$ overnight. Then, cells were washed with PBS, stained with propidium iodide, and analyzed with a BD Accuri C6 flow cytometer (BD, San Jose, CA). For cell apoptosis analysis, the Annexin V-FITC Apoptosis Detection Kit (KEYGEN, Shanghai, China) was used according to the instructions. The apoptosis rate was generated using a BD Accuri C6 flow cytometer (BD Biosciences, Mountain View, CA).

\section{Statistical analysis}

Data in the experiment were analyzed using SPSS software version 16.0 (SPSS, Inc., Chicago, IL). All data are presented as the mean and SD. The significance was determined by Student's $t$-test and one-way ANOVA. A difference of $p<0.05$ was considered statistically significant.

\section{Results}

\section{Downregulation of Inc-SERTAD2-3 expression in OS}

To determine whether lnc-SERTAD2-3 was associated with OS, qPCR was used to detect the expression of lncSERTAD2-3 in OS cell lines and clinical specimens. Levels of lnc-SERTAD2-3 in OS cells (MG-63, U2OS, and Saos-2) were significantly downregulated relative to normal human osteoblasts (hFOB1.19) (Fig. 1A). As expected, decreased lnc-SERTAD2-3 expression, relative to adjacent tissues, was also found in OS specimens (Fig. 1B). In addition, we also found that lower lnc-SERTAD2-3 expression was correlated with an unfavorable prognosis, including tumor size and distant metastasis and recurrence (Table 1). Patients with lower lnc-SERTAD2-3 expression were also associated with a poor overall survival rate (Fig. 1C).

\section{Inc-SERTAD2-3 retarded OS cell growth}

To further investigate the effects of lnc-SERTAD2-3 on OS cells, we elevated lnc-SERTAD2-3 expression in MG-63 and U2OS cells by transfection with a lnc-SERTAD2-3overexpressing plasmid (Fig. 2A). A CCK-8 assay showed that cell growth was inhibited in OS cells due to the overexpression of lnc-SERTAD2-3 (Fig. 2B). Flow cytometry was used to assess changes in the cell cycle and cell apoptosis. The results showed that lnc-SERTAD2-3 induced G1 phase arrest (Fig. 2C) and cell apoptosis (Fig. 2D), which 

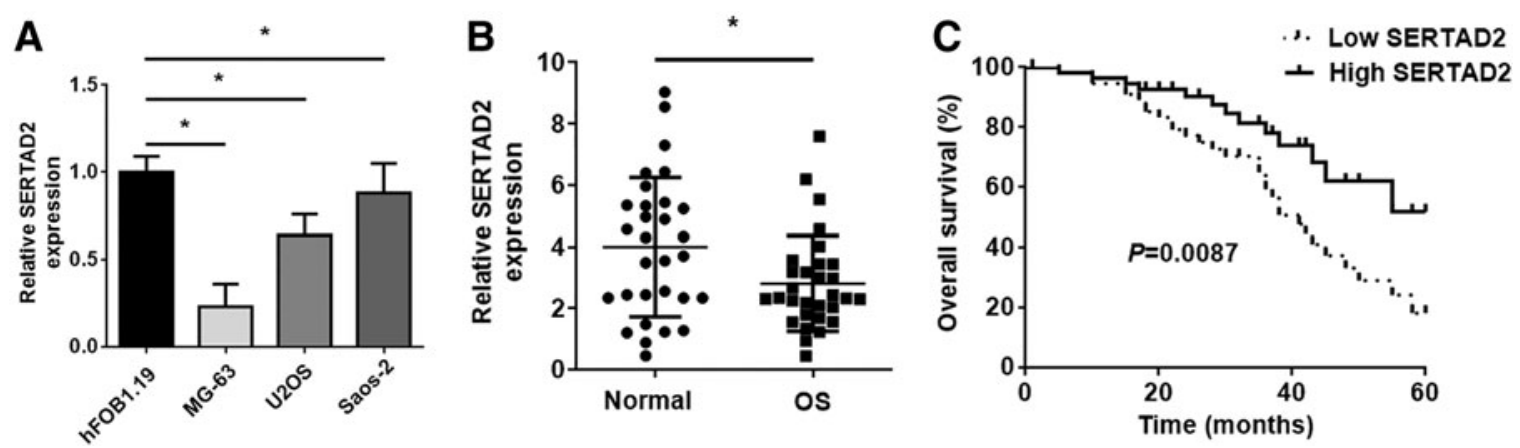

FIG. 1. The expression of 1nc-SERTAD2-3 in OS cell lines and specimens. (A) The expression of 1nc-SERTAD2-3 in hFOB1.19, MG-63, U2OS, and Saos-2 is shown, as examined by qPCR. Experiments were repeated five times. (B) Levels of lnc-SERTAD2-3 in OS tissues and adjacent tissues $(n=30)$, as determined by qPCR. (C) The Kaplan-Meier curve of overall survival rate assessing the correlation of lnc-SERTAD2-3 expression with survival in OS patients $(n=30)$. Data are expressed as the mean $\pm \mathrm{SD}, * p<0.05$. OS, osteosarcoma; qPCR, quantitative real-time PCR.

resulted in inhibition of OS cell growth. In addition, cell invasion, as measured by Transwell assay, indicated that lncSERTAD2-3 suppressed the cells' invasive ability (Fig. 2E).

\section{Inc-SERTAD2-3 acted as a molecular sponge of $\operatorname{miR}-29 \mathrm{c}$}

miRcode was used to predict the potential target miRNA of lnc-SERTAD2-3 and miR-29c was identified. The expression of miR-29c was detected in clinical OS specimens. Interestingly, miR-29c expression and lnc-SERTAD2-3 levels were negatively correlated (Fig. 3A). The expression of miR-29c in OS tissues was significantly higher than that in the adjacent tissues (Fig. 3B). Overexpression of 1nc-SERTAD2-3 significantly suppressed miR-29c levels (Fig. 3C). In addition, levels of the PTEN homolog, a target gene of miR-29c, were

Table 1. The Clinicopathological Characteristics of Osteosarcoma Patients

\begin{tabular}{lcccc}
\hline & & \multicolumn{2}{l}{ lnc-SERTAD2-3 expression } & \\
Group & $\mathrm{n}$ & High & Low & $\mathrm{p}$ \\
\hline Age & & & & \\
$>18$ & 36 & 22 & 14 & 0.8296 \\
$\leq 18$ & 24 & 14 & 10 & \\
Sex & & & & \\
$\quad$ Male & 23 & 12 & 11 & 0.8871 \\
Female & 37 & 20 & 17 & \\
Clinical stage & & & & \\
I/II & 28 & 14 & 14 & 0.2207 \\
III & 32 & 11 & 21 & \\
Size (cm) & & & & \\
$\quad>5$ & 25 & 8 & 17 & $0.0184^{*}$ \\
$\quad \leq 5$ & 35 & 22 & 13 & \\
Distant metastasis & & & \\
$\quad$ Negative & 26 & 18 & 8 & $0.0046^{*}$ \\
Positive & 34 & 11 & 23 & \\
Recurrence & & & & \\
$\quad$ Absent & 23 & 15 & 26 & $0.0088^{*}$ \\
Present & 37 & 11 & & \\
\hline
\end{tabular}

$* p<0.05$. measured by qPCR after transfection of a lnc-SERTAD2-3overexpressing plasmid in OS cells. As expected, the overexpression of lnc-SERTAD2-3 enhanced PTEN expression in OS cells (Fig. 3D). Furthermore, we established a luciferase reporter plasmid containing the wild-type or mutant lnc-SERTAD2-3 sequence for a luciferase assay (Fig. 3E). The luciferase activity following cotransfection with wildtype lnc-SERTAD2-3 and a miR-29c mimic was decreased, but was increased following cotransfection with wild-type lnc-SERTAD2-3 and a miR-29c inhibitor (Fig. 3F).

\section{Reintroduction of miR-29c reversed \\ the effect of Inc-SERTAD2-3 on cells}

To elucidate whether miR-29c affected the role of lncSERTAD2-3 in modulating OS progression, we cotransfected lnc-SERTAD2-3 and a miR-29c mimic in OS cells. The expression of miR-29c and PTEN was verified after cotransfection (Fig. 4A, B). According to the CCK-8 assay, cell growth was significantly enhanced with the restoration of miR-29c in MG-63 and U2OS cells (Fig. 4C). Moreover, flow cytometry revealed that overexpression of miR29c inhibited G1 phase cell cycle arrest and cell apoptosis (Fig. 4D, E) and reversed the lnc-SERTAD2-3-mediated attenuation of the cells invasive capacity (Fig. 4F).

\section{Discussion}

The metastasis and recurrence of OS are the primary reasons for the high death rate of OS (Kager et al., 2017). It is necessary to find novel diagnostic, prognostic, and therapeutic targets for better management. Accumulating studies indicate that lncRNAs are involved in the progression of carcinogenesis for various malignancies, including OS (Smolle and Pichler, 2018; Wang et al., 2018). Therefore, lncRNAs may provide valuable biomarkers for diagnosis, prognosis, and therapy. Our study suggested that lnc-SERTAD2-3 could be a valuable biomarker for patients with OS.

The lnc-SERTAD2-3 gene, located on chromosome 2, expresses a transcript totaling 2928 bp in size. SERTAD genes, considered key nuclear transcriptional players, play a vital role in neuronal cell proliferation, viral infections, and cancer progression (Hsu et al., 2001; Gupta et al., 2003; Zang et al., 2007; Cheong et al., 2009). Recent studies indicate that 
A

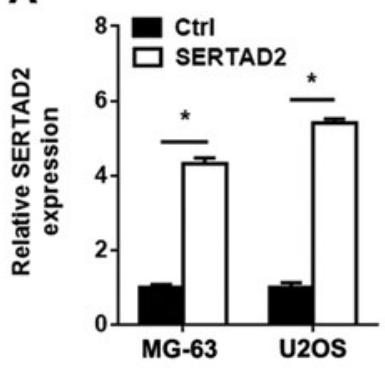

C

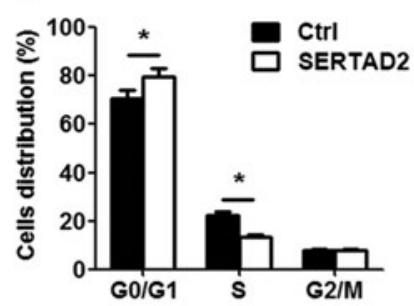

B
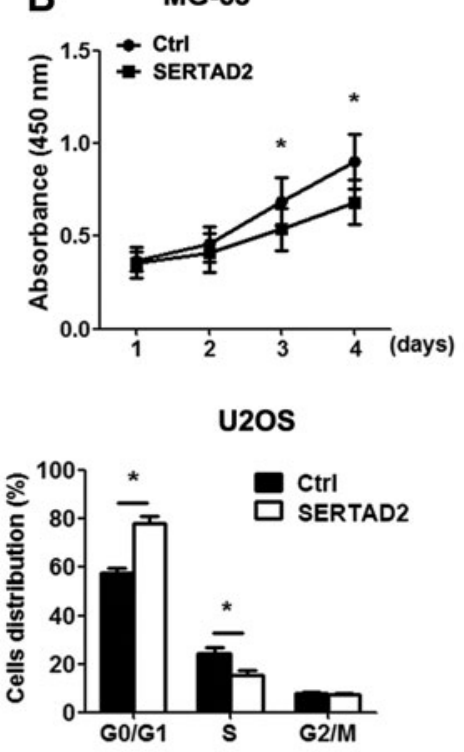

U20s
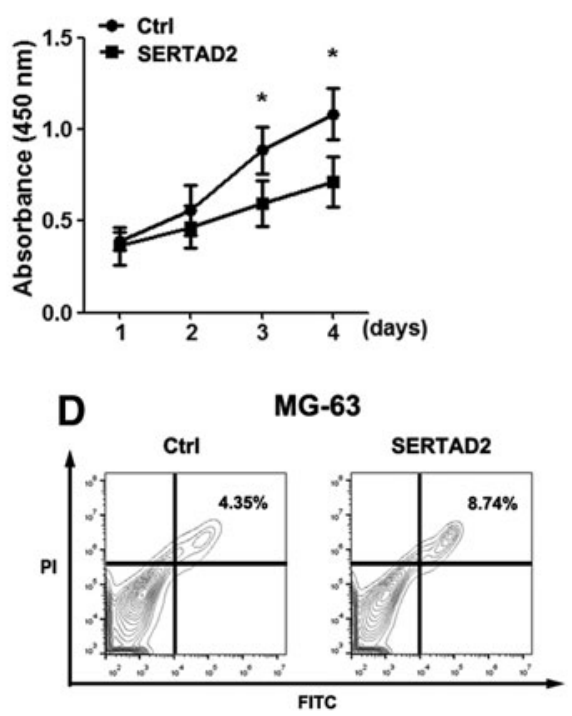
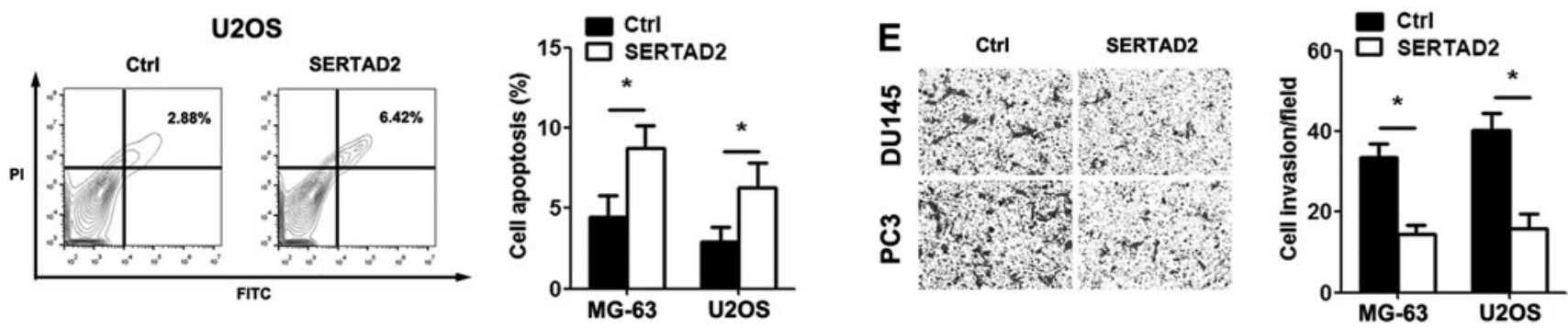

FIG. 2. The effects of lnc-SERTAD2-3 on cell viability, cell cycle, and invasion in OS cells. MG-63 and U2OS cells were transfected with the lnc-SERTAD2-3-overexpressing plasmid (lnc-SERTAD2-3) and control (Ctrl) for $48 \mathrm{~h}$. (A) The expression of lnc-SERTAD2-3 in OS cells after transfection, as assessed by qPCR analysis. (B) Cell viability, as detected by CCK-8 assay, is shown. (C, D) Cell cycle and apoptosis using flow cytometry after transfection. (E) Cell invasive capacity is shown, as evaluated using the Transwell assay. All experiments were repeated five times. $* p<0.05$. CCK-8, Cell Counting Kit-8.

SERTAD members act as transcriptional coregulators to modulate E2F-dependent transcriptional activities (Hsu et al., 2001; Hayashi et al., 2006; Darwish et al., 2007). Accordingly, the SERTAD family is implicated in E2Fmediated cell cycle progression and tumorigenesis. Nevertheless, the role of 1nc-SERTAD2-3 in biological processes remains to be elucidated. In this study, our findings suggested that lnc-SERTAD2-3 might play a critical role in OS carcinogenesis. In addition, lnc-SERTAD2-3 may be a valuable biomarker for OS diagnosis and prognosis. As expected, overexpression of 1nc-SERTAD2-3 in OS cells suppressed cell growth by inducing G1 phase cell cycle arrest and cell apoptosis, suggesting that lnc-SERTAD2-3 may act as a suppressor in the development of OS. Interestingly, a previous study showed that lnc-SERTAD2-3 is an independent prognostic marker of relapse and poor prognosis for epithelial ovarian cancer, and higher lnc-SERTAD2-3 expression is often associated with unfavorable prognosis. These data reveal the distinct prognostic value of lnc-SERTAD2-3 in different cancers (Martini et al., 2017).

Recent studies indicate that aberrant expression of lncRNAs facilitates OS development (Ren et al., 2019; Wu et al., 2020; Yang et al., 2019; Zhang et al., 2019). These
lncRNAs exert their functions by several mechanisms, including targeting some host genes, involvement with signaling pathways and competing with endogenous miRNA. Growing evidence reveals that lncRNAs serve as competitive endogenous RNA for miRNAs, which function as molecular sponges to suppress miRNA expression (Yan et al., 2018; Liao et al., 2019; Su et al., 2019; Wang et al., 2019). Recently, it was indicated that lncRNA exerts its biological function by binding to and "mopping up" miRNA (Chen et al., 2017). miR-29c was identified as a target endogenous miRNA of lnc-SERTAD2-3 using bioinformatics databases. Of note, there was a negative correlation between the expression of lnc-SERTAD2-3 and miR-29c in OS and adjacent tissues, and we demonstrated that lnc-SERTAD2-3 could suppress miR-29c expression by direct binding.

The miR-29 family has been validated in the development and progression of human OS, and upregulation of miR-29c was observed in OS tissue (Hong et al., 2014; Wang et al., 2016; Liu et al., 2019). A previous study showed that miR-29 promotes OS cell proliferation and migration by targeting PTEN, while the targeting of miR-29 exhibits significant cell apoptosis activities in OS (Liu et al., 2019). These results indicate that miR-29, an OS inducer, is involved in OS 


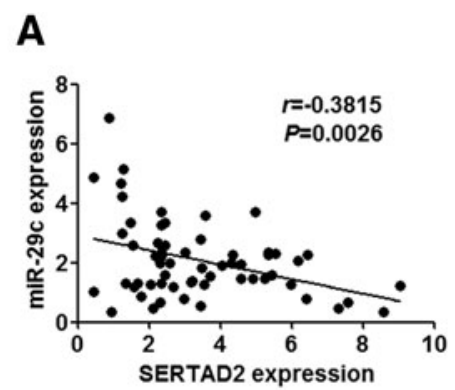

E

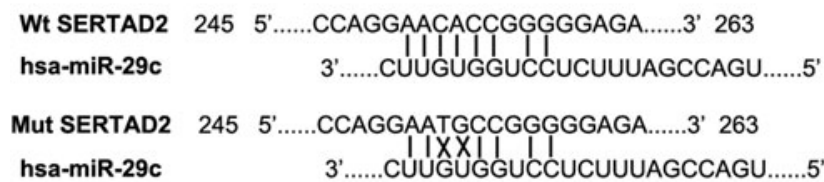

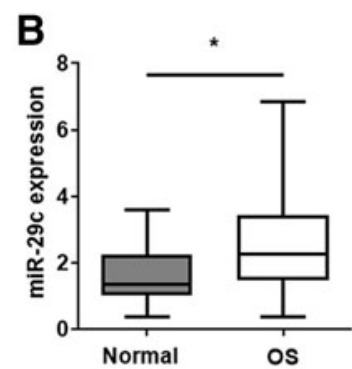
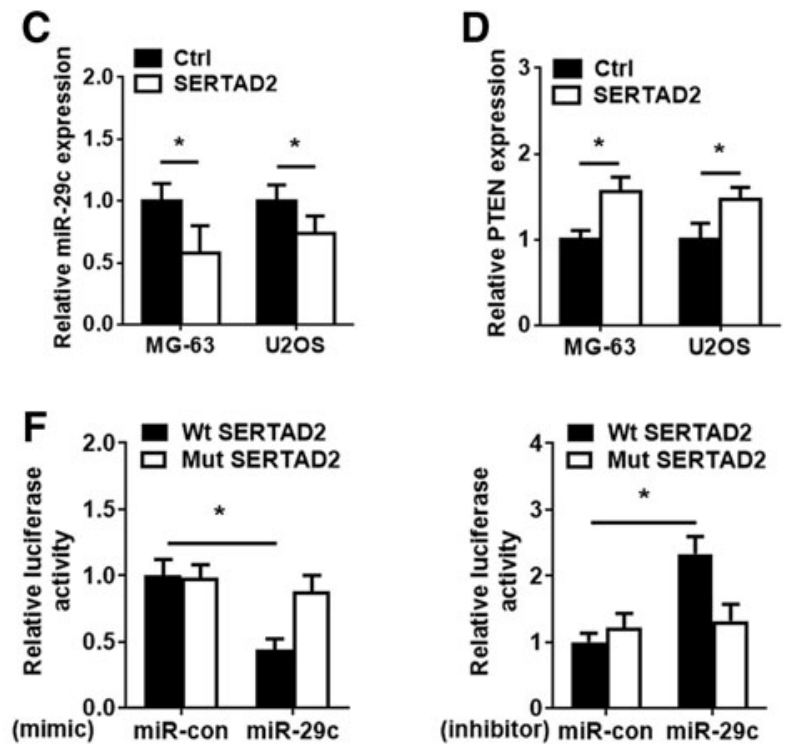

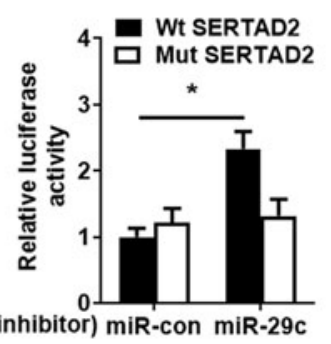

FIG. 3. miR-29c is a target of lnc-SERTAD2-3 in OS. (A) The expression of miR-29c is negatively correlated with lncSERTAD2-3 levels in 60 clinical specimens. (B) The levels of miR-29c in OS tissues and adjacent tissues are shown, as measured by qPCR. (C, D) miR-29c and PTEN expression after transfection with the lnc-SERTAD2-3-overexpressing plasmid (lnc-SERTAD2-3) and the control (Ctrl) for $48 \mathrm{~h}$. (E) The putative wild-type and mutant full-length lnc-SERTAD23 sequence were cloned into a pGL3 luciferase vector. (F) Luciferase activity after cotransfection with the wild-type/mutant luciferase vector and the miR-con/miR-29c mimic. All experiments were repeated five times. ${ }^{*} p<0.05$. PTEN, phosphatase and tensin.

progression and metastasis. Consistently, our study demonstrated that miR-29c promoted OS cell proliferation and invasion, and lnc-SERTAD2-3, by competitively binding to miR-29c, had the opposite effect. As expected, restoration of miR-29c abrogated the antitumor effects of lnc-SERTAD2-3 on cell proliferation, apoptosis, and invasion in OS cells, which confirmed that lnc-SERTAD2-3 exerted its anti-OS effect by binding to miR-29c.

In conclusion, our study demonstrated that lnc-SERTAD23 induced G1 cell cycle arrest and cell apoptosis by the miR$29 \mathrm{c} / \mathrm{PTEN}$ axis in OS and thus may be a valuable biomarker and therapeutic target for OS.
A

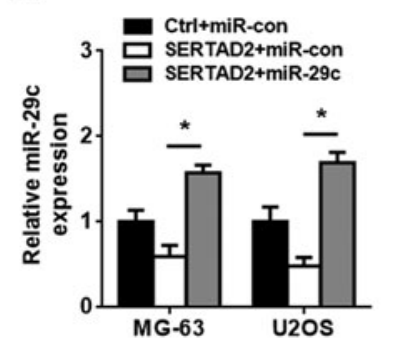

D

MG-63

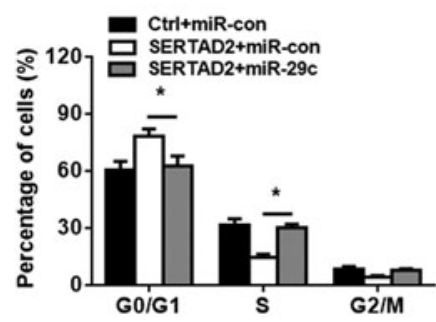

B

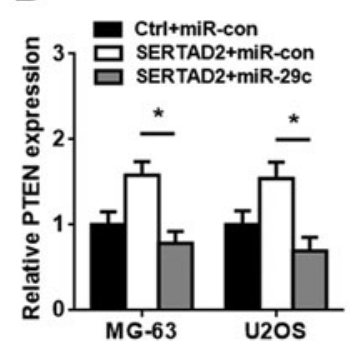

U2OS

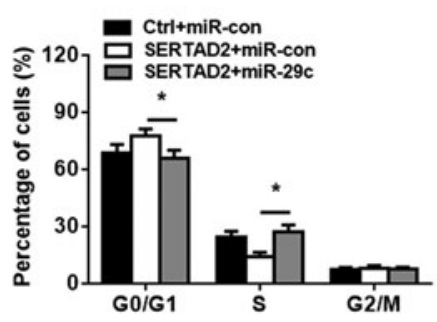

C
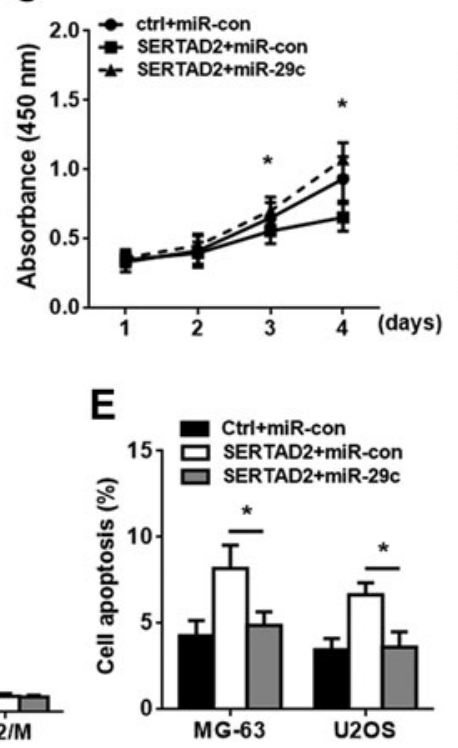

U2OS

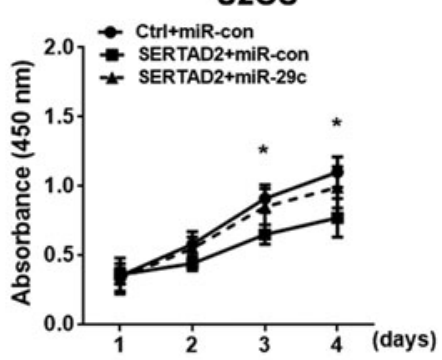

$\mathbf{F}$

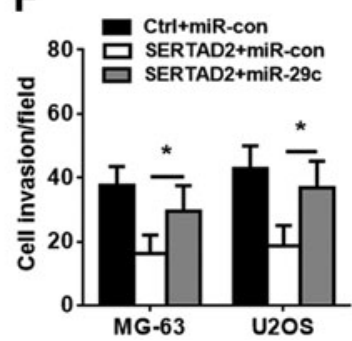

FIG. 4. miR-29c reverses the effect of lnc-SERTAD2-3 on OS cells. MG-63 and U2OS cells were cotransfected with the lnc-SERTAD2-3/Ctrl and the miR-con/miR-29c mimic for $48 \mathrm{~h}$. (A, B) miR-29c and PTEN expression after transfection. (C) CCK-8 assay results detecting cell viability after transfection. (D, E) Cell cycle and apoptosis results using flow cytometry after transfection. (F) Cell invasive capacity using a Transwell assay. All experiments were repeated five times. $* p<0.05$. 


\section{Author Disclosure Statement}

No competing financial interests exist.

\section{Funding Information}

This work was supported by the Beijing Science and Technique Star Foundation (2010B80) and by the National Natural Science Foundation of China (Grant No. 81371911).

\section{References}

Allison DC, Carney SC, Ahlmann ER, et al. (2012) A metaanalysis of osteosarcoma outcomes in the modern medical era. Sarcoma 2012:704872.

Chen R, Wang G, Zheng Y, et al. (2017) Long non-coding RNAs in osteosarcoma. Oncotarget 8:20462-20475.

Cheong JK, Gunaratnam L, Zang ZJ, et al. (2009) TRIP-Br2 promotes oncogenesis in nude mice and is frequently overexpressed in multiple human tumors. J Transl Med 7:8.

Darwish H, Cho JM, Loignon M, Alaoui-Jamali MA (2007) Overexpression of SERTAD3, a putative oncogene located within the 19q13 amplicon, induces E2F activity and promotes tumor growth. Oncogene 26:4319-4328.

de Oliveira JC, Oliveira LC, Mathias C, et al. (2019) Long noncoding RNAs in cancer: another layer of complexity. J Gene Med 21:e3065.

Flippot R, Beinse G, Boileve A, et al. (2019) Long non-coding RNAs in genitourinary malignancies: a whole new world. Nat Rev Urol 16:484-504.

Gupta S, Takhar PP, Degenkolbe R, et al. (2003) The human papillomavirus type 11 and 16 E6 proteins modulate the cellcycle regulator and transcription cofactor TRIP-Br1. Virology 317:155-164.

Hayashi R, Goto Y, Ikeda R, et al. (2006) CDCA4 is an E2F transcription factor family-induced nuclear factor that regulates E2F-dependent transcriptional activation and cell proliferation. J Biol Chem 281:35633-35648.

Hong Q, Fang J, Pang Y, Zheng J (2014) Prognostic value of the microRNA-29 family in patients with primary osteosarcomas. Med Oncol 31:37.

Hsu SI, Yang CM, Sim KG, et al. (2001) TRIP-Br: a novel family of PHD zinc finger- and bromodomain-interacting proteins that regulate the transcriptional activity of E2F-1/ DP-1. EMBO J 20:2273-2285.

Kager L, Tamamyan G, Bielack S (2017) Novel insights and therapeutic interventions for pediatric osteosarcoma. Future Oncol 13:357-368.

Li Z, Dou P, Liu T, He S (2017) Application of long noncoding RNAs in osteosarcoma: biomarkers and therapeutic targets. Cell Physiol Biochem 42:1407-1419.

Liao S, Xing S, Ma Y (2019) LncRNA SNHG16 sponges miR98-5p to regulate cellular processes in osteosarcoma. Cancer Chemother Pharmacol 83:1065-1074.

Liu Q, Geng P, Shi L, et al. (2019) miR-29 promotes osteosarcoma cell proliferation and migration by targeting PTEN. Oncol Lett 17:883-890.

Martini P, Paracchini L, Caratti G, et al. (2017) lncRNAs as novel indicators of patients' prognosis in stage I epithelial ovarian cancer: a retrospective and multicentric study. Clin Cancer Res 23:2356-2366.

Mongre RK, Jung S, Mishra CB, et al. (2019) Prognostic and clinicopathological significance of SERTAD1 in various types of cancer risk: a systematic review and retrospective analysis. Cancers (Basel) 11. DOI: 10.3390/cancers11030337.
Ottaviani G, Jaffe N (2009) The epidemiology of osteosarcoma. Cancer Treat Res 152:3-13.

Ren Z, Hu Y, Li G, et al. (2019) HIF-1alpha induced long noncoding RNA FOXD2-AS1 promotes the osteosarcoma through repressing p21. Biomed Pharmacother 117:109104.

Smolle MA, Pichler M (2018) The role of long non-coding RNAs in osteosarcoma. Noncoding RNA 4. DOI: 10.3390/ ncrna4010007.

Su P, Mu S, Wang Z (2019) Long noncoding RNA SNHG16 promotes osteosarcoma cells migration and invasion via sponging miRNA-340. DNA Cell Biol 38:170-175.

Sun H, Huang Z, Sheng W, Xu MD (2018) Emerging roles of long non-coding RNAs in tumor metabolism. J Hematol Oncol 11:106.

Wang C, Jing J, Cheng L (2018) Emerging roles of non-coding RNAs in the pathogenesis, diagnosis and prognosis of osteosarcoma. Invest New Drugs 36:1116-1132.

Wang CY, Ren JB, Liu M, Yu L (2016) Targeting miR-29 induces apoptosis of osteosarcoma MG-63 cells via regulation of TGF-beta1/PUMA signal. Eur Rev Med Pharmacol Sci 20: $3552-3560$.

Wang X, Zou J, Chen H, et al. (2019) Long noncoding RNA NORAD regulates cancer cell proliferation and migration in human osteosarcoma by endogenously competing with miR199a-3p. IUBMB Life 71:1482-1491.

Wu S, Gu Z, Wu Y, et al. (2020) LINC00324 accelerates the proliferation and migration of osteosarcoma through regulating WDR66. J Cell Physiol 235:339-348.

Yan L, Wu X, Liu Y, Xian W (2018) LncRNA Linc00511 promotes osteosarcoma cell proliferation and migration through sponging miR-765. J Cell Biochem. DOI: 10.1002/ jcb.27999.

Yang Q, Yu H, Yin Q, et al. (2019) lncRNA-NEF is downregulated in osteosarcoma and inhibits cancer cell migration and invasion by downregulating miRNA-21. Oncol Lett 17: 5403-5408.

Zang ZJ, Sim KG, Cheong JK, et al. (2007) Exploiting the TRIP-Br family of cell cycle regulatory proteins as chemotherapeutic drug targets in human cancer. Cancer Biol Ther 6: 712-718.

Zhang H, Lu Y, Wang J, et al. (2019) Downregulation of the long noncoding RNA FOXD2AS1 inhibits cell proliferation, migration and invasion in osteosarcoma. Mol Med Rep 20: 292-302.

Address correspondence to: Xuesong Zhang, PhD Department of Orthopaedics The PLA General Hospital Beijing 100000 China

E-mail: zhangxuesong301@ outlook.com

Rui Zhang, MS

Department of Emergency Medicine Xiang'an Hospital of Xiamen University School of Medicine Xiamen University Xiamen 361102 China

E-mail: erlisanda@163.com 